\title{
THE HARD X-RAY REFLECTION ON COLD MATTER
}

\author{
E. JOURDAIN AND J.P. ROQUES \\ CESR, BP4946, 31029 Toulouse, Cedex, France
}

\section{INTRODUCTION}

We have simulated the reflection on cold matter $(1,2,3 \& 5)$ in a variety of situations to determine which informations can actually be inferred from observations. We modelled a semi-infinite plane parallel medium of solar abundance matter (4), semi-isotropically illuminated by a $\mathrm{X} / \gamma$ ray source. The spectra are calculated from a Monte-Carlo method without any approximation in the cross-sections. $\Theta$ is the angle over which the reflecting matter is seen $\left(90^{\circ}=\right.$ face-on), $\Theta=$ all means a spatially integrated spectrum. Fref is the ratio of the reflected over direct component.

\section{DISCUSSION}

The presence of a reflected component introduces a hump between 10 and $500 \mathrm{keV}(1,2,3)$. More precisely, our study has shown that:

* small values of $\Theta$, Fref or $\mathrm{Nh}\left(10^{-24} \mathrm{~cm}^{-2}\right)$ can explain the no detection of this phenomenon even when cold matter is present in the vicinity of the source.

* we can obtain rather identical spectra for several couples (Fref, $\Theta$ ) ( fig.1).

* the 2 breaks occur at $10 \mathrm{keV}$ and $30 \mathrm{keV}$ almost independently of the parameter values. However, the 2nd slope change is smooth and can mimic a thermal law. Moreover, a comptonized primary spectrum may hidde this feature.

* between 30 and $60 \mathrm{keV}$, the composite (direct+reflected) spectrum seems to have a slope close to that of the primary one. A deficit of photons occurs below and above this energy range.

* above $30 \mathrm{keV}$, the reflection effect is hard to interpret due to the curved shape of the spectrum. A broken power law fit will give results depending on the energy band chosen.

\section{CONCLUSION}

It is very likely that cold matter and reflection ${ }_{\mathrm{h}}^{\mathrm{P}}$ exist in AGNs central regions. It is thus crucial o to get data from 2 to $\sim 60 \mathrm{keV}$ with the same $\mathrm{t}$ instrument and a good sensitivity to determine o how the spectrum is affected and deduce $n$ informations on the source environment. / However, as Fref and $\Theta$ act similarly, their $k$ values will be generally model dependent. The primary spectrum must also be investigated.

\section{References:}

1)George I.\&Fabian A., 1991,MNRAS,249,352

2)Guibert P.\&Rees M.,1988,MNRAS, 233, 475

3)Lightman A.\& White T., 1988, APJ, 335, 57

4)Morrisson \& McCammon,1983,APJ,270, 119

5)Pounds K. A.et al., 1990, NATURE, 44, 132

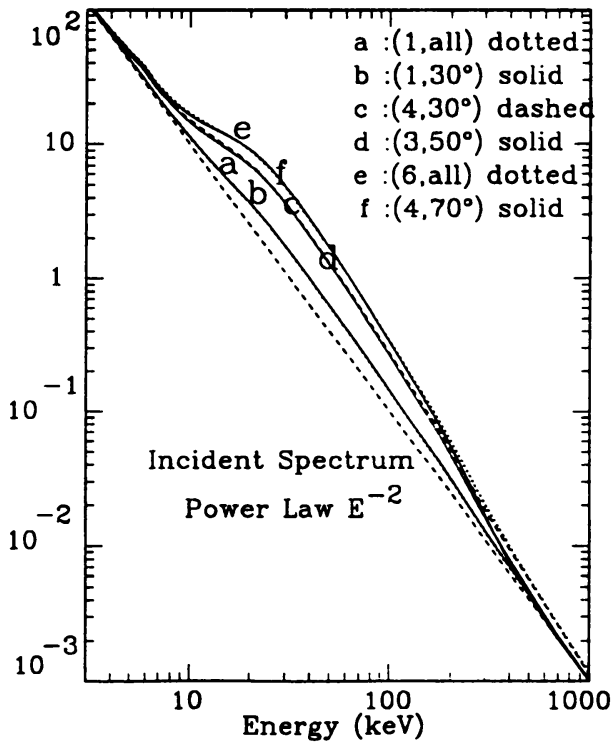

Fig.1: Spectra obtained for different couples (Fref, $\Theta$ ) as indicated for each label 